\title{
Le statut professionnel des illustrateurs pour la jeunesse : une lente et difficile conquête
}

The Professional Status of Children's Illustrators: A Slow and Difficult Struggle

\section{El estado profesional de los ilustradores de libros para jóvenes: una conquista lenta y difícil}

\section{Françoise Lepage}

Volume 51, numéro 2, avril-juin 2005

Les métiers du livre au Québec

URI : https://id.erudit.org/iderudit/1030089ar

DOI : https://doi.org/10.7202/1030089ar

Aller au sommaire du numéro

Éditeur(s)

Association pour l'avancement des sciences et des techniques de la documentation (ASTED)

ISSN

0315-2340 (imprimé)

2291-8949 (numérique)

Découvrir la revue

Citer cet article

Lepage, F. (2005). Le statut professionnel des illustrateurs pour la jeunesse : une lente et difficile conquête. Documentation et bibliothèques, 51(2), 79-88. https://doi.org/10.7202/1030089ar
Résumé de l'article

Les illustrateurs pour la jeunesse ont d'abord été des illustrateurs de presse peu soucieux du public pour lequel ils dessinaient. Les Éditions Jeunesse ont été les premières à s'intéresser à la qualité de l'image dans les années 1960, mais le véritable épanouissement de cet art s'est manifesté avec le renouveau de l'album dans les années 1980. Malgré la fondation d'une association d'illustrateurs en 1983 et l'établissement de prix et de divers incitatifs, les illustrateurs québécois n'ont toujours pas atteint la reconnaissance professionnelle qui leur est due. Chroniquement sous-payé, le travail de l'illustrateur est aujourd'hui concurrencé par l'ordinateur, les banques d'images et la photographie.
Tous droits réservés (c) Association pour l'avancement des sciences et des techniques de la documentation (ASTED), 2005
Ce document est protégé par la loi sur le droit d'auteur. L’utilisation des services d’Érudit (y compris la reproduction) est assujettie à sa politique d'utilisation que vous pouvez consulter en ligne. 


\title{
Le statut professionnel des illustrateurs pour la jeunesse : une lente et difficile conquête
}

\author{
FRANÇOISE LEPAGE \\ Lettres françaises \\ Université d'Ottawa \\ fralepage@yahoo.com
}

\begin{abstract}
RÉSUMÉ | ABSTRACTS | RESUMEN
Les illustrateurs pour la jeunesse ont d'abord été des illustrateurs de presse peu soucieux du public pour lequel ils dessinaient. Les Éditions Jeunesse ont été les premières à s'intéresser à la qualité de l'image dans les années 196o, mais le véritable épanouissement de cet art s'est manifesté avec le renouveau de l'album dans les années 1980. Malgré la fondation d'une association d'illustrateurs en 1983 et l'établissement de prix et de divers incitatifs, les illustrateurs québécois n'ont toujours pas atteint la reconnaissance professionnelle qui leur est due. Chroniquement sous-payé, le travail de l'illustrateur est aujourd'hui concurrencé par l'ordinateur, les banques d'images et la photographie.
\end{abstract}

\section{The Professional Status of Children's Illustrators:} A Slow and Difficult Struggle

Children's illustrators began as publisher's illustrators with little regard for the public for whom they were illustrating works. The Editions Jeunesse were amongst the first to be interested in the quality of illustrations in the 1960 . However, the real development of this art form became evident in the 1980 sith the renewed interest in children books. In spite of the creation of an association of illustrators in 1983 and the creation of a prize and other incentives, Québec illustrators are still not recognised as professionals. Chronically underpaid, the illustrator competes with the computer, image banks and photography.

\section{El estado profesional de los ilustradores de libros para} jóvenes: una conquista lenta y difícil

En un principio, los ilustradores de literatura juvenil trabajaron en la prensa y no se preocuparon mucho por el público para el cual dibujaban. En la década de los años 6o, las Éditions Jeunesse (Ediciones Juveniles) fueron las primeras en interesarse por la calidad de la imagen, pero el verdadero florecimiento de esta forma artística se manifestó con la renovación del álbum ilustrado en la década de los años 80 . A pesar de la fundación de una asociación de ilustradores en 1983 y la creación de premios $y$ diversos estímulos, los ilustradores quebequenses siguen sin lograr el reconocimiento profesional que se merecen. La labor del ilustrador, que cobra crónicamente una paga por debajo de lo normal, compite actualmente con la computadora, los bancos de imágenes y la fotografía.

1. À ce jour, on a peu écrit sur l'illustration pour la jeunesse et sur les illustrateurs. Mentionnons Françoise Lepage (2000 : 443-512), dans le chapitre intitulé «La séduction de l'image», qui trace un historique de l'illustration pour la jeunesse au Québec. De la même auteure, «L'image dans l'album pour enfants : enquête sur une libération» (2003b : 21-44). Francine Sarrasin (1991) a publié et présenté le catalogue d'une exposition d'illustrations d'albums pour enfants et, à partir de 1993, a supervisé la publication d'une série d'analyses d'images dans la revue Lurelu.
Q UI DIT LIVRE POUR LA JEUNESSE dit illustration. Les images aident les apprentis lecteurs, les lecteurs peu compétents ou les non-lecteurs à comprendre le texte jusque vers 10 ans. Aussi est-on bien fondé de supposer que l'illustrateur représente un acteur incontournable du champ de la littérature pour la jeunesse. Pourtant, la reconnaissance, dans le domaine du livre, de ces artistes qui s'expriment, non pas avec des mots et des phrases, mais selon un autre système sémiotique composé de lignes, de formes et de couleurs, ne va pas de soi. Cette particularité du mode d'expression de l'illustrateur constitue un facteur de marginalisation dans le monde de l'écrit. En outre, ces artistes travaillent "pour la jeunesse", public bien précis mais lui aussi marginal et qui n'est pas toujours considéré à sa juste valeur. Enfin, si les illustrateurs de livres pour adultes sont généralement des artistes visuels bien connus du public, qui illustrent les grandes œuvres de la littérature et qui font l'objet d'études, d'articles ou de thèses, les illustrateurs pour la jeunesse travaillent sur une littérature «marginalisée » qui ne bénéficie guère de la reconnaissance publique ${ }^{1}$.

Il nous a donc semblé opportun de nous interroger sur le statut professionnel de l'illustrateur pour la jeunesse. Comment s'est établie cette profession au cours de son histoire? Quelle perception les divers intervenants du monde du livre ont-ils de l'illustrateur? Cette perception a-t-elle évolué depuis les origines de la littérature québécoise pour la jeunesse en 1920 ? L'art de l'illustration constitue-t-il une activité professionnelle reconnue comme telle? Depuis quand? Voilà autant de questions que le présent article tente d'éclairer en présentant à la fois une perspective diachronique du statut des illustrateurs pour la jeunesse et un bilan de la situation de ces «faiseurs d'images».

Cette question du statut des illustrateurs n'ayant jamais été abordée, la recherche fondamentale de documents et de témoignages restait à faire. Les données historiques utilisées dans cet article sont extraites de divers fonds d'archives, dans lesquels elles se trouvent disséminées. Précisons que ces 
informations sont rares, le travail des illustrateurs ayant suscité peu de documents écrits. Encore plus clairsemés, les commentaires des critiques littéraires n'aident pas à se former une idée de ce que les professionnels du livre et le public pensaient de l'illustration du livre pour la jeunesse dans les débuts. Ce n'est guère que depuis une dizaine d'années que la critique se risque parfois à dire un mot sur les illustrations. Enfin, l'étude de la situation actuelle des illustrateurs est fondée sur des témoignages de personnes œuvrant dans le domaine, recueillis au cours de lété 2004.

\section{UNE NAISSANCE LABORIEUSE (1920-1949)}

Quand la littérature pour la jeunesse voit le jour en 1920, lors du lancement de la revue L'Oiseau bleu par la Société Saint-Jean-Baptiste de Montréal (SSJBM), l'image a déjà un long passé didactique, qui a été étudié, tant en France qu'au Québec, et sur lequel nous ne reviendrons pas ici. Qu'il suffise de renvoyer aux études sur les méthodes utilisées par les Jésuites surtout, mais aussi par d'autres communautés enseignantes, dans des buts de pédagogie ou de conversion religieuse (Gagnon, 1975; Adhémar, 1981). D'autres congrégations, en particulier les Franciscains, ont publié avant 1920 des ouvrages joliment illustrés, probablement par des membres de la communauté, qui s'acquittaient de cette tâche de façon anonyme (Lepage, 2003a).

Les premiers illustrateurs laïques, ceux qui ont mis en images les Contes historiques de la SSJBM, venaient d'horizons divers. Certains, comme Rita Mount, Maurice LeBel, Jean-Baptiste Lagacé ou Claire Fauteux, étaient des artistes bien connus de leur temps. Tous, à l'exception de Rita Mount, étaient engagés dans des activités professionnelles à plein temps : postes d'enseignement à la Commission des écoles catholiques de Montréal ou à l'Université Laval de Montréal ou encore copiste d'archives pour l'ambassade du Canada à Paris. D'autres, comme Louis-Joseph Dubois, Napoléon Savard, Samuel Brodeur ou Georges Latour, étaient des illustrateurs de presse à une époque où la photographie n'était pas encore couramment utilisée dans les journaux car, la reproduction des clichés étant coûteuse, elle aurait fait augmenter excessivement le prix de vente des quotidiens (Lepage, 200o : 447). Pour garder les prix à des niveaux plus populaires, les journaux faisaient appel à des dessinateurs qui reproduisaient des photographies : portraits, paysages ou événements d'actualité. Cette expérience professionnelle de "copistes» déteint sur la conception que ces artistes se faisaient de l'illustration. Celle-ci reste très proche du texte, se veut réaliste et documentaire, tout comme les illustrations de journaux. L'apport de l'imaginaire y est très faible, d'autant plus que la révolution surréaliste vient tout juste de se produire, les manifestes d'André Breton étant publiés à partir de
1924. Le roman historique, qui domine la production des années 1920-1940, se prête bien à cette forme d'illustration documentaire. Le plus connu des illustrateurs des origines, James McIsaac, qui a notamment illustré la plupart des romans de Marie-Claire Daveluy, a commencé sa carrière comme dessinateur au service des Travaux publics de la Ville de Montréal, avant de devenir inspecteur des incendies. Son activité d'illustrateur s'est étendue de 1919 à 1945 et, dans ses temps libres, McIsaac faisait également œuvre d'artistepeintre (Lepage, 1991).

Le procès-verbal de la réunion du 10 mars 1921 de la SSJBM nous apprend que les illustrateurs des Contes historiques étaient rémunérés pour leur travail. Ainsi, Onésime Léger perçoit 15 \$ pour la mise en images du conte historique intitulé "Les précurseurs ", c'est-à-dire pour une série de 12 vignettes en quadrichromie. Exceptionnellement, le 8 octobre 1935, le comité de la revue L'Oiseau bleu vote « une prime à Louis-Joseph Dubois » pour le soin qu'il accorde à ses dessins, mais le montant n'en est pas précisé. Ces illustrateurs déjà actifs dans les années 1920 se sont formés sur le tas ou ont suivi des cours au Conseil des arts et manufactures. Fondé en 1872, cet établissement avait pour objectif de former non pas des artistes, mais des dessinateurs pour les besoins du monde industriel. Les illustrateurs des générations subséquentes ont pu suivre les cours dispensés dans les Écoles des beauxarts de Montréal et de Québec, mises sur pied respectivement en 1921 et 1923. On voit donc qu'à l'origine de la littérature pour la jeunesse les illustrateurs pratiquent cet art d'autant plus occasionnellement que la production littéraire n'est pas très abondante et il est probable qu'aucun d'entre eux ne se serait défini comme «illustrateur».

Odette Fumet-Vincent (1911-1995) est la première artiste à avoir fait profession d'illustratrice. Française d'origine, elle avait suivi des cours de dessin pendant trois ans à l'Académie Julian à Paris, avec l'intention de se diriger vers l'illustration. Elle commence sa carrière en ce domaine en 1934, en France, puis continue après son installation au Québec, en 1937, jusque dans les années 1970. Elle a laissé une œuvre graphique très abondante en littérature pour la jeunesse - une bonne centaine de titres -, tout en collaborant avec son mari, Rodolphe Vincent, illustrateur commercial, pour lequel elle préparait les planches à photograver. Tous deux ont enseigné le dessin chez les frères de l'Instruction chrétienne. En 1952, ils ont mis sur pied un cours de dessin par correspondance, La gerbe, offert jusque dans les années 1960.

À cette époque, antérieure à la Révolution tranquille, le travail des illustrateurs est constamment limité par les consignes d'économie qu'imposent les éditeurs ou les techniques de reproduction - dessins en noir et blanc ou au nombre limité de couleurs, impossibilité de faire réduire ou agrandir les dessins - 
ainsi que par la censure sévère qu'exercent les membres du clergé responsables de journaux ou de maisons d'édition. Le moindre trait de crayon trop «suggestif» sur le devant d'un pantalon ou la moindre tenue trop décontractée suffisait à faire rejeter le dessin (Turgeon, 1970).

Contrairement à ce qui s'est passé dans l'illustration du livre pour adultes, où un grand nombre d'artistes de renom ont donné à leur pratique ses lettres de noblesse - on pense ici à Georges Delfosse (Florence de Rodolphe Girard), Clarence Gagnon (Maria Chapdelaine de Louis Hémon), Edwin Holgate (Metropolitan Museum de Robert Choquette), Alfred Pellan (Les Îles de la nuit d'Alain Grandbois) et beaucoup d'autres -, peu d'artistes connus ont apporté une contribution à l'illustration pour la jeunesse des origines. Dans cette catégorie, on retient Jean Paul Lemieux, qui avait un moment envisagé une carrière d'illustrateur (Bernier, 1990: 81) et qui n'a illustré que deux livres pour la jeunesse, dont Le Petit Page de Frontenac de Maxine. Citons encore Louis Archambault et Jacques de Tonnancour (L'Aiglon blanc des Illinois de Maxine), Henri Beaulac, dont l'apport s'est limité à un bandeau dans la biographie La Vie gracieuse de Catherine Tekakwitha (1934) de Juliette Lavergne, et Émile LaPalme, illustrateur des Éditions Parizeau (Michon, 2004; Bernier, 1991), à qui l'on doit les images de Ristontac (1945), conte d'Andrée Maillet que l'on aurait depuis longtemps oublié s'il n'avait été illustré par cet artiste hors du commun. L'album pour très jeunes lecteurs n'existant pas, l'illustration se présente le plus souvent, dans les romans, sous la forme de dessins d'une page en noir et blanc. Ristontac inaugure la lignée des livres de contes destinés aux jeunes lecteurs de l'école primaire avec des illustrations en couleurs. C'est aussi le seul livre pour enfants illustré par LaPalme.

Il convient de souligner que les possibilités techniques, à la fois limitées et coûteuses, de reproduction des couleurs, jointes à l'exiguïté du marché francophone, ne favorisent pas alors la production de beaux livres pour la jeunesse. Il existe des livres abondamment illustrés en couleurs, comme les brochures de la collection «Gloires nationales» du frère Guy Laviolette (pseudonyme de Michel-Henri Gingras), mais la palette chromatique est toujours limitée à quatre couleurs.

Si l'illustration de livres pour adultes connaît une grande effervescence dans les années 1920 à 1950, il n'en est pas de même dans l'illustration pour la jeunesse, où, à une ou deux exceptions près, l'illustration est confiée à des personnes qui ne pratiquent cette activité qu'accessoirement. La plupart de ces illustrateurs d'occasion sont des artistes commerciaux qui ne se préoccupent guère de la spécificité du public pour lequel ils dessinent. Se présentant la plupart du temps sous la forme de modestes dessins à la plume en noir et blanc, l'illustration n'a alors rien pour attirer le regard du public. Comme le signale Silvie Bernier (1990: 108) :

«On fait [...] peu de cas des décorateurs de livres qui ont consacré une bonne partie de leur carrière au travail d'illustrateur. C'est le cas d'Henri Julien, Robert LaPalme, Cécile Chabot, Albert Fournier, Henri Beaulac, Jean Simard, Françoise Bujold. Tous ces faiseurs d'images ont souvent une crédibilité réduite dans le milieu artistique et on signale rarement leur contribution à l'histoire de l'art. Pourtant, bien que leur imagerie ne soit pas toujours innovatrice, ces illustrateurs ont certainement favorisé la diffusion des nouvelles tendances artistiques en utilisant un médium facilement accessible au grand public.»

En fait, on peut dire que le métier d'illustrateur n'existe pas encore à la fin des années 1940. Comme la sculpture qui prend forme progressivement sous les coups de burin du sculpteur, l'illustration commence tout juste à sortir de sa gangue.

\section{L'ÉMERGENCE D'UNE PROFESSION (1950-1970)}

À la fin des années 1950 et dans les années 1960, devant la difficulté que rencontre la littérature québécoise pour la jeunesse à percer dans un marché envahi par les importations de livres étrangers, en l'occurrence français, les artisans du livre décident de passer à l'action. Béatrice Clément, elle-même auteure pour la jeunesse, fonde en 1948 l'Association des écrivains pour la jeunesse, dont l'objectif, comme son nom l'indique, est essentiellement littéraire : faire connaitre les écrivains pour la jeunesse au public québécois, améliorer la qualité de la production littéraire et développer chez les jeunes le goût de la lecture. Pour ce faire, l'Association crée une coopérative d'édition - Les Éditions Jeunesse - dirigée par Daniel Lareau, peintre et illustrateur. Les membres qui désirent publier dans cette coopérative doivent présenter des manuscrits répondant à trois critères établis par le comité de lecture : la qualité de la langue, la pertinence du contenu pour le lecteur et la valeur de lillustration (Labelle, $1949:$ 301). C'est la première fois qu'un document témoigne ouvertement d'une préoccupation pour la qualité de l'illustration.

Bien que l'Association ne survive que six ans, elle met toujours de l'avant cette nécessité d'une cohabitation harmonieuse du texte et de l'image. Dans un article anonyme de La Presse (5 juillet 1950) qui présente les buts de l'Association, le journaliste écrit :

"[L'Association] veut fournir [aux jeunes] des livres de qualité, spécialement adaptés à leur âge, leurs goûts et leur mentalité. Du même coup, elle veut faire connaître les écrivains qui 
consacrent leur talent à léducation des jeunes tout aussi bien que créer un lien entre les auteurs et les illustrateurs pour réaliser des publications de premier choix. »

Simultanément, dans les années 1948-1949, Daniel Lareau s'associe avec Béatrice Clément pour produire une série de bandes dessinées intitulée Parade historique qui reprend l'esprit des Contes historiques de la SSJBM, mais dans une facture plus moderne. Le fonds Béatrice-Clément des Archives nationales du Québec à Montréal (ANQM) contient quelques lettres qui permettent de se faire une idée du projet et des conditions de travail des deux partenaires. Écrivaine et illustrateur s'engagent à fournir aux journaux intéressés une page par semaine pendant 84 semaines, temps nécessaire pour raconter la vie des 42 personnages inscrits sur leur liste. Ils offrent aux journaux les flans d'imprimerie pour 15 \$ la page, «c'est-à-dire au même prix que les États-Unis nous vendent leurs comics» (D. Lareau, ANQM, S3, SS4, D3, 20 novembre 1948) ${ }^{2}$. Ils décident de partager à parts égales les profits et les pertes encourus par la publication de leur œuvre à la fois dans les journaux et en albums (ANQM, 4-47-5, 28 octobre 1949). Le Petit Journal, publication hebdomadaire de Montréal, donne 15 \$ "pour chaque épisode d'une page de format tabloïd [...] si ledit épisode est publié en noir et blanc et une somme de 25 \$ si ledit épisode est publié en couleurs» (ANQM, 4-47-4, 13 janvier 1949). Le journal The Ensign de Montréal, publié par Campion Press, achète les droits de publication pour un montant hebdomadaire de $5 \$$ versés à Daniel Lareau, pendant les 6 premiers mois, tandis que Béatrice Clément conserve les droits de traduction et, par la suite, la part de l'illustrateur doit être augmentée à 7,50 \$ par semaine (ANQM, 4-47-5, 11 mai 1949).

Après la disparition prématurée de cette première association d'auteurs pour la jeunesse, Béatrice Clément n'abandonne pas l'idée de faire un nouvel essai. En 1959, un petit groupe d'écrivains et de personnes intéressées se réunissent à la librairie Dussault pour discuter de littérature pour la jeunesse et de la formation d'une nouvelle association. Après de nombreux ajournements, Béatrice Clément ressuscite les Éditions Jeunesse et demande à Réal d'Anjou, directeur des Éditions du Pélican, d'en prendre les rênes. Son cousin, le jésuite Marie-Joseph d'Anjou, se charge de la révision linguistique et de l'orthodoxie morale et Paule Daveluy, qui est, à cette époque, une romancière à succès, devient directrice de collection. Fait à noter, la nouvelle maison d'édition se réserve les services d'une conseillère artistique, Simone HudonBeaulac, chargée de veiller à la qualité de l'illustration (Lepage, 2003 : 119-132), tout en souhaitant aussi la voir collaborer en tant qu'illustratrice à la collection «Panache» (ANQSF, lettre de Réal d'Anjou à MarieJoseph d'Anjou, 6 mars 1963). Cette collaboration n'aura jamais lieu, Simone Hudon n'ayant illustré que ses propres recueils de comptines. Malgré tout, le comité d'honneur de la maison d'édition, formé de personnalités éminentes, soit le cardinal Paul-Émile Léger, $\mathrm{M}^{\mathrm{gr}}$ Albert Tessier, $\mathrm{M}^{\mathrm{gr}}$ Félix-Antoine Savard, mère Gérin-Lajoie, Alvine Bélisle et Marie-Claire Daveluy, ne comporte aucun artiste, indice que le métier d'illustrateur n'est pas encore reconnu.

Le rôle de Simone Hudon consiste, selon ses propres termes, «à découvrir et à guider les artistes désireux d'illustrer des ouvrages écrits pour les jeunes» (Gauthier, 1962: 22). Elle précise qu'il n'existe pas encore de tradition de l'illustration pour la jeunesse, qu'il faut la créer et que cette activité comporte deux volets : l'art de mettre en images la pensée d'un écrivain et l'adaptation au public cible. La conseillère artistique souligne en outre l'importance de donner aux jeunes de beaux livres qui puissent contribuer à leur formation intellectuelle, morale et esthétique, d'où la nécessité d'attirer de bons artistes dans le domaine de l'illustration : "Plusieurs croient se diminuer en illustrant pour les jeunes; nous devons combattre ce préjugé, qui nuit à léclosion des talents cachés chez les jeunes» (Ibid. : 22). D'ailleurs, les Éditions Jeunesse choisissent comme mot d'ordre une phrase d'Astrid Lindgren, auteure suédoise de Fifi Brindacier : «Le plus exquis de ce qu'il y a de meilleur». Pour parvenir à cet objectif, elles préparent une brochure qui parait en 1962 et se vend o,50 \$. Intitulée Nos ouvres sont aussi votre ouvrage, elle contient des conseils pour les auteurs et les illustrateurs. Pour ces derniers, il est précisé que :

\section{"Les dessins illustrent vraiment; il ne leur suffit pas d'orner. Non seulement ils traduisent en images le texte de l'écrivain (tel passage, telle scène avec tous ses détails), mais, par leur facture, ils en adoptent et en manifestent l'esprit.»}

Il est également demandé aux illustrateurs de connaître la psychologie des jeunes, de se tenir au courant de la littérature pour la jeunesse et de la critique "afin de savoir ce qui plaît ou déplaît, forme et déforme, et pourquoi» ainsi que de connaître les exigences techniques de l'impression pour ne pas causer d'inutiles frais supplémentaires à l'éditeur (APPD, dossier Éditions Jeunesse).

En 1963, lors des audiences de la Commission d'enquête sur le commerce du livre dans la province de Québec (Commission Bouchard), les Éditions Jeunesse présentent un mémoire rédigé par le père d'Anjou. Il y parle de l'importance d'avoir une littérature pour la

2. Pour donner une idée du coût de la vie au début de la Seconde Guerre mondiale, mentionnons qu'en 1940 Le Progrès du Saguenay annonçait des pantoufle pour dames au prix de $0,75 \$$, des chaussures habillées pour 1,49 \$, une livre de beurre pour 0,26 \$, une douzaine d'œufs pour 0,29 \$ et une Plymouth pour 904 \$. 
jeunesse d'ici et accorde une certaine place à l'illustration. Il mentionne qu'en France de grands artistes s'intéressent à l'illustration pour la jeunesse. Pourquoi pas au Québec?

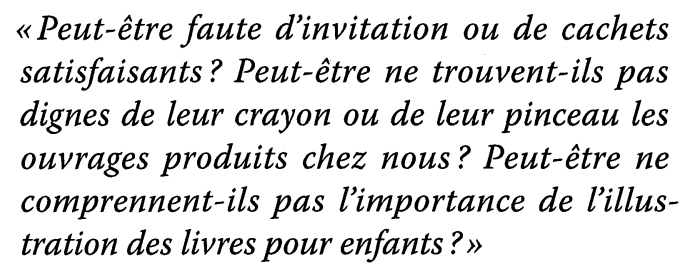

APPD, D’Anjou, $1963: 6$.

Le mémoire souligne également le caractère formateur d'une illustration de qualité, qui aide les jeunes à prendre conscience de leurs propres aptitudes pour le dessin et à les cultiver. Il ne fait pas de doute que, pour les Éditions Jeunesse, il y a une bonne façon. d'illustrer un livre pour enfants :

"La bonne manière consiste à éclairer de couleurs vives, contrastées sans être choquantes, le dessin conçu. Ou, si le dessin n'a pas de couleurs, à le composer de telle sorte que le personnage ou l'objet principal accroche l'oil et invite l'enfant à chercher dans le texte tout ce que suggère l'illustration.»

$$
\text { Ibid. : } 7 \text {. }
$$

Cette conception d'une illustration «spéciale» pour enfants sera battue en brèche par les pourfendeurs européens et américains de l'album traditionnel, comme François Ruy-Vidal et Harlin-Quist, pour qui il n'existe pas de bonnes images "pour enfants", mais seulement des images de qualité capables de séduire tous les publics.

Toute l'histoire des Éditions Jeunesse, du moins telle qu'elle apparaît dans les documents d'archives, est marquée par de nombreuses difficultés, mais surtout par l'apparente rareté des illustrateurs compétents. Le comité éditorial a préparé un questionnaire non daté, mais que l'on peut situer entre 1962 et 1964, qu'il adresse aux illustrateurs désireux de travailler pour les Éditions Jeunesse. Il se lit comme suit :

«1. Avez-vous le goût et vous arrive-t-il de lire des albums pour tout-petits, des ouvrages destinés à des jeunes de 9 à 13 ans, des livres pour adolescents?

2. Nommez:

a. Quelques collections dont l'esprit vous plaît. Dites pourquoi.

b. Vos illustrateurs favoris pour les jeunes. Sont-ils canadiens?

c. Trois titres d'albums (livres) que vous reliriez avec plaisir.

3. Jacques Gagnier (1918-1978) s'était surtout fait connaitre comme décorateur des célèbres Fridolinades. À partir des années 1950, il dirige un atelier de productions publicitaires à Montréal. d. Le nom de l'auteur et de l'illustrateur de chacun de ces ouvrages.

3. Dans les trois livres mentionnés plus haut (2c), l'artiste a-t-il rendu justice à l'écrivain? Justifiez votre réponse. (S'il s'agit de livres épuisés ou introuvables en bibliothèque, envoyez l'ouvrage avec votre réponse).

4. Avez-vous déjà illustré des livres? Lesquels?

5. Pour quel âge de lecteurs désirez-vous illustrer des ouvrages?

6. Pourquoi les livres canadiens ne sont-ils pas, en général, aussi bien illustrés que les livres français ou belges? Pourquoi nos livres sont-ils rarement illustrés en couleurs?

7. En 12 lignes (maximum), dites quelles exigences on doit satisfaire, quels défauts on doit éviter dans les dessins exécutés pour les enfants de 6 à 10 ans :

a. du point de vue esthétique?

b. du point de vue psychologique?

c. du point de vue religieux et moral?

Adressez vos réponses au R.P. d'Anjou, s.j., [...], avec un mandat ou chèque de 3 \$. Vous recevrez un commentaire de votre travail et une deuxième série de questions. "

ANQM, 4-53-1.

Cette «consultation» vise, on le voit, à établir si le postulant connaît un peu les livres pour la jeunesse, s'il a réfléchi à la spécificité de cette forme de création - dans ses rapports avec le texte, en particulier - et à celle du public cible. Béatrice Clément se charge des entrevues avec les artistes, «entrevues d'ailleurs infécondes parce que lesdits artistes ne sont pas "au point" ". (ANQSF, lettre de Réal d'Anjou à Marie-Joseph d'Anjou, 30 novembre 1962)

Des lettres de Marie-Joseph d'Anjou à Paule Daveluy donnent des précisions sur les difficultés rencontrées :

«Notre gros problème [aux Éditions Jeunesse]: mettre en branle les dessinateurs. M.d'Anjou va embaucher un de ses amis de Québec [...]. M Mle Clément vient d'approcher M. Jacques Gagnier, qui a paru s'intéresser vivement à nos projets [...]. Quelle acquisition, celle de Gagnier! Lui et son équipe (car il a une équipe de dessinateurs) pourraient illustrer les albums de "Cocarde blanche"."

APPD, 19 août $1961^{3}$.

Sept ans plus tard, le 27 juin 1968, le père d'Anjou écrit à Paule Daveluy: "Vous ne soupçonnez pas la difficulté qu'il y a présentement à trouver un illustrateur convenable» (APPD), et on pourrait multiplier les exemples. 
S'il est si difficile de trouver de bons illustrateurs, c'est aussi parce que la rémunération qui leur est concédée pour leur travail est dérisoire. Le 30 novembre 1963, France Bédard, lillustratrice qui a été chargée d'illustrer Mon missel du dimanche, reçoit 300 \$ et 3 exemplaires gratuits pour l'exécution de son contrat, lequel comprenait la réalisation de 12 illustrations pleine page, 17 illustrations d'une demi-page, 5 motifs, 23 petits dessins et un dessin de couverture. Aussi ne faut-il pas s'étonner si, le 17 mars 1964 , lorsque l'éditeur Réal d'Anjou lui propose d'illustrer un livre de prières, la jeune illustratrice - elle a alors 19 ans - décline l'invitation. Elle allègue avoir été naïve d'accepter l'illustration du missel dans les conditions fixées et que, si elle tient compte du temps qu'elle a consacré au travail et aux inévitables retouches, sa rémunération s'élève à un $1,10 \$ /$ l'heure. Le père d'Anjou lui offrant 150 \$ à 200 \$ pour l'illustration $\mathrm{du}$ livre de prières, l'illustratrice termine en disant : «Croyez-vous pouvoir, avec cette somme, payer un artiste pour dessiner 29 dessins, dont 2 en couleurs?» (ANQSF, 17 mars 1964)

Le 14 février 1964, Gabriel Perreault demande 15 \$ la page pour faire la séparation de couleurs de l'album Samuel de Champlain qu'il a illustré; l'éditeur lui en offre $10 \$$, le 22 février. Le comité des Éditions Jeunesse est parfaitement conscient de la médiocrité des rémunérations offertes aux illustrateurs, comme en témoigne cette remarque de l'éditeur luimême, déclarant que les illustrations de Malmenoir le mauvais par Étienne Gauthier sont «très convenables et [... que l'illustrateur] pourrait, sans être extravagant, exiger deux fois autant et même plus.» (ANQSF, lettre de Réal d'Anjou à Béatrice Clément, 19 juillet 1963)

Dans les années 1950 et 1960 , la pression qu'exerçait la bande dessinée américaine a certainement contribué au développement d'une illustration made in Quebec. Les religieux qui dirigeaient les maisons d'édition, de même que les enseignants et les animateurs des loisirs des jeunes, trouvaient la bande dessinée américaine immorale. Pour lui faire obstacle, certains éditeurs se dotent d'illustrateurs qu'il est possible d'encadrer et de contrôler. Dans ce contexte, les éditions Fides embauchent Maurice Petitdidier, en 1953, pour illustrer leurs publications pour la jeunesse et, en 1957, l'artiste commence à produire des bandes dessinées pour la revue Hérauts. Ce changement est toutefois de courte durée. Les coûts de production de bandes dessinées locales sont si élevés et la charge de travail si lourde, pour un seul illustrateur, que Fides revient aux bandes dessinées américaines en septembre 1960 (Hébert, 1982 : 115).

Simultanément, à la fin des années 1950 et au début des années 1960, Leméac publie une série d'adaptations de contes traditionnels dans des albums grand format, avec des illustrations en couleurs signées $\mathrm{Al}$ Ros, qui s'adressent aux élèves de l'école primaire. La route s'ouvre à la production de livres dans lesquels l'image prend de plus en plus d'importance. Il faudra cependant attendre 1966 pour que paraisse le premier album moderne pour tout-petits, dans lequel limage occupe plus d'espace que le texte : Un drôle de petit cheval d'Henriette Major, illustré par Guy Gaucher.

Après 1960, l'art sort de son ghetto et se popularise. La différence entre arts dits majeurs et arts mineurs tend à s'effacer et cette époque voit la résurgence des métiers d'art, qui permettent au grand public de s'offrir des objets où prime la qualité esthétique. L'illustration du livre pour la jeunesse et plus particulièrement des grands albums publiés par Leméac participe de cette démocratisation de l'art : "La Révolution tranquille n'est pas seulement la mise sur pied d'institutions publiques, elle est aussi et surtout l'introduction d'un rapport "public" à la vie sociale et culturelle» (Fournier, $1986: 80$ ). Si les artistes bénéficient grandement de cette ouverture, les illustrateurs pour la jeunesse restent tributaires du manque de reconnaissance de la littérature pour laquelle ils travaillent et qui, à la fin des années 1960, est au bord de la disparition.

\section{L'ALBUM MODERNE ET L'APOTHÉOSE DE L'ILLUSTRATION (1970-)}

Au début des années 1960, un mouvement se dessine qui valorise l'importance de mettre sous les yeux des enfants des images de qualité ${ }^{4}$. Les enseignants des matières artistiques, dont le statut n'est toujours pas reconnu, joignent leurs voix au concert, comme le prouve ce témoignage d'une éducatrice d'enfants handicapés :

"La qualité esthétique et la valeur du matériel
didactique est (sic) le seul moyen de former
l'émotion esthétique et de lutter contre l'enva-
hissement de la pacotille. Tout ce qui s'offre
à la perception visuelle devrait être choisi en
fonction de la qualité de la forme et de la co-
loration.» AUQAM, 5/10 : 31 .

Malgré ces prises de position des professionnels de l'enseignement pour faire valoir l'importance de la qualité dans léducation visuelle, il reste beaucoup de chemin à parcourir. L'ouverture de la littérature pour la jeunesse aux non-lecteurs, c'est-à-dire à ceux qui ont le plus besoin d'images, aurait dû susciter immédiatement de belles perspectives d'avenir pour les illustrateurs. Malheureusement, cette littérature vit des années difficiles après 1965, date à laquelle la suppression des prix scolaires de fin d'année fait dramatiquement baisser les ventes de livres québécois et affecte tout le cycle de la production. En 1970, moins de cinq livres pour la jeunesse sortent des presses. Devant la disparition imminente de ce secteur culturel, les forces se mobilisent sous la bannière de Communication-Jeunesse, organisme de promotion 
fondé en 1971 par Paule Daveluy et Suzanne CloutierRocher (Lepage, 2000 : 275). Guy Gaucher, designer et réalisateur à Radio-Canada qui fait partie du premier conseil d'administration de Communication-Jeunesse, a eu la bonne idée de proposer pour l'organisme un nom qui évoque plus que la seule communication écrite :

"Nous vivons, insistait-il, à lère de la communication, de l'interdisciplinarité. Le livre, par ses illustrations et par l'utilisation de la diapositive, du disque, de la marionnette, du théâtre, de la vidéo, de la télévision, devient une ouvre visuelle, auditive, en même temps que littéraire.»

Cloutier-Rocher, 1997 : 59.

À cette époque, la mode est à l'audiovisuel, dont on débat des avantages et des inconvénients, et la relance des écrits pour la jeunesse monopolise toute l'attention des participants au colloque Création culturelle pour la jeunesse et identité québécoise, tenu en novembre 1972. Les illustrateurs sont absents de cette grande manifestation de mise au point, ou, du moins, n'interviennent-ils pas.

La création des éditions Le Tamanoir, en 1974, qui deviendront La courte échelle en 1978, marque un tournant dans l'illustration de l'album pour les petits, type de livre le plus apte à mettre en valeur le talent des illustrateurs. Bien que quelques albums de facture moderne aient été publiés avant 1974, comme Ouram, d'Anne Vallières, et La Poulette grise, illustrée par Louise Méthé, tout change dans la deuxième moitié des années 1970, quand Le Tamanoir fait appel aux services de jeunes créateurs qui renouvellent l'aspect du livre québécois. Dans la foulée de cet essor considérable de la demande en illustration contemporaine, un groupe d'illustrateurs et d'artistes visuels se rencontrent en 1982 pour fonder l'Association des illustrateurs et illustratrices du Québec (AIIQ), officiellement enregistrée le 27 février 1983. L'AIIQ a été mise sur pied pour "promouvoir l'art de l'illustration et faire en sorte que la profession dillustrateur se pratique dans les meilleures conditions possibles» (AIIQ, 19981999 : VIII). Pour ce faire, l'Association a publié divers documents, dont un Code de tarification et un Contrat de commande et de licence qui "permettent aux membres de prendre connaissance de leurs droits et d'assurer une diffusion à leurs ouvres " (AIIQ, 19981999 : VIII). Depuis quelques années, l'AIIQ organise à Montréal une Biennale de l'illustration, exposition qui permet de faire connaître le travail des illustrateurs. Dans la version 2004 de la Biennale, une soixantaine d'artistes étaient exposés, dont un grand nombre illustrent pour la jeunesse. Dans l'article qu'elle consacre aux associations d'éditeurs, Josée Vincent note que :

«L'apparition des associations professionnelles est une étape importante du processus d'auto- nomisation du champ littéraire. Pour qu'elles puissent voir le jour, il faut d'abord que le champ soit à peu près constitué, et cela présuppose qu'il regroupe une quantité suffisante d'agents.»

Vincent, $2001: 547$.

Elle souligne également que la constitution d'une association est liée à l'augmentation de la production et à l'existence d'une situation potentiellement menaçante pour l'exercice de la profession. Selon Suzanne Duranceau, fondatrice de l'AIIQ :

"On considérait souvent que publier les images d'un artiste à côté d'un texte était presque lui faire un cadeau. On lui faisait de la publicité, disait-on. On nous faisait parfois sentir que si on n'était pas content, il y avait des dizaines d'artistes qui attendaient derrière la porte, et c'était pour des sommes dérisoires. J'ai vu des livres pour enfants pour lesquels on payait 35 \$ la pleine page couleur [...] On jouait sur le fait que les artistes aiment leur art, qu'ils font cela pour le plaisir et qu'ils n'ont pas besoin d'être payés.»

ESD : 3 .

En 1981, les prix du Conseil des Arts du Canada, devenus prix du Gouverneur général en 1987, s'enrichissent de deux sections, l'une pour l'illustration, l'autre pour la traduction. Ce prix décerné annuellement à un illustrateur permet de mettre son ouvre sous les feux de la rampe et d'améliorer les ventes de l'album primé. Depuis 1986, la Commission du droit de prêt public dédommage les créateurs de livres, dont les illustrateurs, pour les emprunts en bibliothèques. Toutes ces initiatives témoignent d'un intérêt accru pour le travail des illustrateurs, mais ne suffisent pas à améliorer réellement leur situation. En effet, le nombre d'illustrateurs talentueux qui font leur marque dans l'illustration n'a cessé de s'accroître depuis les années 1980 :

«Le marché québécois est saturé d'illustrateurs de talent et l'AIIQ en est un peu responsable, car nous avons suscité un engouement formidable pour l'illustration en faisant la promotion du travail des illustrateurs, en étant présents dans les médias pour le faire connaître et reconnaître. Dans un sens, nous avons noyé notre propre marché qui était trop petit pour une telle abondance d'artistes.»

ESD : 4 .

Si La courte échelle publie moins d'albums qu'à ses débuts, d'autres maisons d'édition, comme Les 400 coups, Dominique et Compagnie ou Banjo, ont pris la relève et produisent des livres d'images de grande qualité. L'album pour enfants est devenu, de nos jours, une pièce maîtresse de la littérature pour la jeunesse, certainement la plus connue et la plus 
spectaculaire. Devant une telle floraison d'œuvres remarquables, il a semblé pertinent d'interroger les intéressés, c'est-à-dire les illustratrices et illustrateurs du Québec, pour mieux connaître leurs conditions de travail et la perception qu'ils ont de leur statut.

\section{MINI-ENQUÊTE SUR LES ILLUSTRATEURS CONTEMPORAINS}

Pour les besoins de cet article, 19 illustrateurs québécois ont reçu un questionnaire par courrier électronique (voir annexe). Sept d'entre eux ont répondu, soit un taux de réponse de $37 \%$. La compilation des réponses et commentaires permet de voir que la très grande majorité des répondants ont reçu une formation générale en arts visuels (cégep ou université). Un seul se dit totalement autodidacte. La plupart (5/7) n'ont acquis aucune formation particulière en illustration pour la jeunesse; deux ont étudié sous la direction d'un illustrateur professionnel. Ils sont unanimes à déclarer que la meilleure formation est encore la pratique professionnelle. Tous ont d'autres activités professionnelles, parallèlement à leur travail d'illustrateurs pour la jeunesse : autres genres d'illustration (éditoriale, commerciale, publicitaire), bandes dessinées, enseignement ou ateliers de création, peinture de chevalet, graphisme, décors de théâtre, etc.

Le temps consacré à l'illustration pour la jeunesse est très variable, oscillant entre $25 \%$ et $90 \%$, les pourcentages plus élevés étant généralement atteints par les illustrateurs les plus connus et les plus prolifiques. D'une façon générale, le pourcentage de revenu retiré du travail en illustration pour la jeunesse est inférieur d'au moins $10 \%$ au pourcentage de temps investi. Ainsi, un illustrateur qui consacre $60 \%$ de son temps au livre pour la jeunesse en retire $50 \%$ de ses revenus, celui qui donne $25 \%$ de son temps à cet art lui doit environ $10 \%$ de ses revenus, etc.

L'unanimité se fait encore sur les questions 8 et 9 du questionnaire. Les illustrateurs sont unanimes à dire qu'au Québec - et cette précision est importante - il est impossible ou très difficile de vivre en ne faisant que de l'illustration de livres pour la jeunesse, l'édition générale et l'édition scolaire étant très mal payées. Une dizaine d'illustrateurs, peut-être, arrivent à vivre en pratiquant leur art dans ce contexte. Seuls les contrats commerciaux (publicité, emballages) sont rentables. Tous les répondants s'entendent pour dire que les tarifs pratiqués au Québec ne permettent généralement pas à un illustrateur de vivre, sauf s'il a beaucoup de contrats et s'il travaille vite et bien. La plupart des illustrateurs doivent absolument se tailler une place sur le marché canadien-anglais (Toronto) ou américain.

Les conditions d'existence de nombreux illustrateurs sont difficiles : aucune sécurité d'emploi, pas d'avantages sociaux, travail ininterrompu, sans soirées ni week-ends libres, sans vacances, pour pouvoir absorber les périodes sans contrats. Pour un des répondants, le salaire d'un illustrateur équivaut à peu près à celui d'un employé d'une chaîne de restauration rapide.

Enfin, les réponses sont plus divergentes en ce qui concerne la valorisation de l'illustration dans l'opinion publique. Trois répondants mentionnent que le travail des illustrateurs pour la jeunesse est mal connu, que la valorisation est nulle ou moyenne ou qu'elle importe peu. Un répondant considère que l'illustration demeure un domaine peu valorisé, rarement reconnu comme un art, qui s'est fait damer le pion par la bande dessinée, beaucoup plus appréciée de nos jours. Enfin, deux répondants soulignent le fait que le public apprécie et valorise beaucoup plus l'art de l'illustration que le milieu de l'édition. Certains parents rêvent de voir leur enfant doué pour le dessin devenir illustrateur. Quant aux principaux «consommateurs» de l'image, en l'occurrence les enfants rencontrés par ces répondants dans les écoles ou les ateliers, ils sont déçus d'apprendre que les illustrateurs sont obligés de vivre très modestement et «ne roulent pas en Cadillac ».

Cette mini-enquête met en évidence plusieurs faits. Premièrement, même si le nombre d'albums publiés au Québec a augmenté considérablement depuis une trentaine d'années, les illustrateurs de livres pour la jeunesse contemporains ne reçoivent généralement pas de formation spécialisée dans leur domaine d'activité. Ils acquièrent une formation générale en arts visuels, puis se forment sur le tas. Deuxièmement, ils pâtissent des difficultés inhérentes au monde de l'édition dans un milieu où la concurrence est forte et le marché restreint. Troisièmement, l'illustration pour la jeunesse est un travail exigeant, mal rémunéré, dont «les tarifs n'ont pas bougé depuis 25 ans» (ESD : 4). Il demande non seulement beaucoup de talent et d'imagination, mais aussi beaucoup de temps, la moindre modification pouvant entrainer plusieurs heures de travail. Dans ces conditions, le temps investi est colossal par rapport à la médiocrité de la rémunération. Quatrièmement, devant les joyaux, que d'aucuns n'hésitent pas à qualifier de « véritables livres d'art», produits par les illustrateurs, on est étonné de voir que la valorisation n'est pas tellement perçue par les créateurs de ces images. Faute de pouvoir ou de vouloir rémunérer correctement les illustrateurs, les intervenants du monde de l'édition n'ont pas intérêt à trop valoriser leur travail. Par contre, si le public semble plus réceptif, les illustrateurs ont l'impression d'être peu connus. Ils se trouvent logés à la même enseigne que les auteurs pour la jeunesse, souvent considérés comme des écrivains de seconde zone dont on ne retient même pas le nom. Il est tout de même choquant de constater que, dans le domaine du livre pour la jeunesse, qui constitue, à lui seul, environ un 
tiers des ventes en librairie, les créateurs sont les plus mal payés.

Dans une entrevue publiée tout récemment dans L'actualité, l'illustratrice Ninon Pelletier ne se montre pas très optimiste :

«Notre art est menacé. Les publications ont de plus en plus recours aux banques d'images offertes sur le Web. Certains directeurs artistiques s'en remettent exclusivement à la photo, d'autres pensent qu'on peut simproviser illustrateur.»

$$
\text { Cayouette, } 2004: 10 .
$$

Suzanne Duranceau abonde dans le même sens. Elle déplore que le public n'accorde pas beaucoup de valeur aux images puisque, avec les banques d'images, les appareils photos numériques, la compétition avec les photos d'art travaillées à l'ordinateur, la démocratisation des loisirs artistiques, tout le monde a accès à des milliers d'images ou peut en faire soi-même. La notion d'artiste se banalise au point qu'on ne fait plus la différence entre amateurs et professionnels. Les jeunes se tournent plus volontiers vers la création d'images informatisées (production de sites Internet, de jeux, de disques compacts) que vers l'illustration traditionnelle, "qui est considérée sur le marché comme presque archaïque et muséologique». (ESD : 5)

\section{ENCORE BEAUCOUP À FAIRE...}

Ce survol de l'évolution du statut de l'illustrateur pour la jeunesse montre que la profession s'est développée très lentement, à la suite de la littérature pour la jeunesse, elle-même marginalisée par rapport à la littérature générale. À ses débuts, l'illustration est souvent considérée par les éditeurs comme un mal nécessaire, auquel il faut bien sacrifier, mais sans encourir de trop grands frais. Lillustration est alors le fait de dessinateurs de presse ou d'artistes amateurs ou professionnels qui ne pratiquent cet art que de façon accidentelle. C'est à l'Association des écrivains pour la jeunesse et, surtout, aux Éditions Jeunesse que revient le mérite de s'être préoccupées de la spécificité de lillustration destinée aux jeunes, de la qualité de l'illustration et de la formation des illustrateurs. Mais déjà, à cette époque, la rémunération dérisoire accordée au travail des illustrateurs n'attire pas beaucoup d'artistes dans ce métier. Avec le développement de l'édition pour la jeunesse dans les années 1980, le développement de la technique qui permet une reproduction plus facile et plus fidèle de la couleur, l'album pour les petits connaît une expansion sans précédent. Pour se protéger des abus, les illustrateurs se regroupent en une association professionnelle, mais les conditions particulières d'exiguïté du marché du livre québécois ne leur permettent toujours pas de vivre de leur art, d'autant plus que les nouvelles technologies informatiques et numériques font désormais concurrence aux illustrations traditionnelles. Il reste encore beaucoup à faire pour reconnaitre, au sein du système de production du livre, le rôle social du livre pour la jeunesse et de ses artisans, qu'il s'agisse des écrivains ou des illustrateurs, et pour accorder à ces derniers le statut qui leur revient, celui d'artistes à part entière. $(\uparrow$

\section{ANNEXE}

\section{Sondage sur le statut des illustrateurs de livres pour la jeunesse (27-29 mai 2004)}

1. Où avez-vous acquis votre formation artistique (cégep, universités, écoles d'arts, stages, cours particuliers, autodidacte)?

2. Les établissements que vous avez fréquentés étaient-ils situés au Québec, au Canada, dans un pays étranger?

3. Avez-vous reçu une formation particulière dans le domaine de l'illustration du livre en général et de l'illustration du livre pour la jeunesse en particulier?

4. Combien d'ouvrages pour la jeunesse avez-vous illustrés?

5. Exercez-vous d'autres activités professionnelles en plus de l'illustration de livres pour enfants? Lesquelles?

6. Quel pourcentage approximatif de votre temps de travail consacrez-vous à l'illustration de livres pour la jeunesse (la moitié, le tiers, etc.)?

7. Quel pourcentage de vos revenus professionnels retirez-vous de lillustration de livres pour la jeunesse?

8. Considérez-vous que votre travail d'illustrateur est correctement rémunéré?

9. Est-il possible, pour un illustrateur québécois, de vivre en ne faisant que de l'illustration pour la jeunesse?

10. Estimez-vous que votre travail d'illustrateur est suffisamment valorisé dans l'opinion publique?

\section{Liste des illustratrices et illustrateurs contactés}

Barrette, Doris

Beshwaty, Steve

Egger, Virginie

Franson, Leanne

Laliberté, Mario

Langlois, Suzanne

Levert, Mireille

Lino

Melanson, Luc

Merola, Caroline 


\section{Myotte, Elsa \\ Ouellet, Joanne \\ Parent, Raymond \\ Pratt, Pierre \\ Reno, Alain \\ Roberts, Bruce \\ Sarrazin, Marisol \\ St-Aubin, Bruno \\ Villeneuve, Anne}

\section{SOURCFS CONSULTÉES}

\section{Sources manuscrites}

Archives nationales du Québec à Montréal (ANQM), fonds BéatriceClément, p. 43.

Archives nationales du Québec à Sainte-Foy (ANQSF), fonds Éditions Jeunesse, p. 744, non classé.

Archives de l'Université du Québec à Montréal (AUQAM), fonds Irène-Senécal, $10 \mathrm{p}$.

Archives personnelles de Paule Daveluy (APPD), non classées.

Entrevue avec Suzanne Duranceau (ESD), réalisée par courriel, le 21 juillet 2004.

Société Saint-Jean-Baptiste de Montréal. Procès-verbaux des réunions de la Société.

\section{Sources imprimées}

Adhémar, Jean. 1981. «L'enseignement par l'image». Gazette des beaux-arts (septembre) : 52-60.

Association des illustrateurs et illustratrices du Québec. 1998-1999. Répertoire. Montréal, L'Association. 227 p.

Bernier, Silvie. 1990. Du texte à l'image. Le livre illustré au Québec. Sainte-Foy, Les Presses de l'Université Laval. 335 p.

Cayouette, Pierre. 2004. «Le crayon de Ninon». L'actualité (15 mai), $\mathrm{n}^{0} 29(8): 10$.

Cloutier-Rocher, Suzanne: 1997. «La naissance de CommunicationJeunesse ». Lurelu, $\mathrm{n}^{\circ} 20$ (1) : 58-60.

Éditions Jeunesse. 1962. Nos œuvres sont aussi votre ouvrage. $44 \mathrm{p}$.
Fournier, Marcel. 1986. Les Générations d'artistes. Québec, Institut québécois de recherche sur la culture. $202 \mathrm{p}$.

Gagnon, François-Marc. 1975. La Conversion par l'image. Montréal, Bellarmin. $141 \mathrm{p}$.

Gauthier, François. 1962. D'où viennent les livres pour notre jeunesse? Le samedi (novembre) : 11 et 22.

Hébert, François. 1982. Hérauts, première véritable revue de bandes dessinées québécoises. La Nouvelle Barre du jour (110-111) : 113-120.

Labelle, Jean-Paul. 1949. «Écrivains pour la jeunesse». Relations 107 (novembre) : 301.

Lepage, Françoise. 1991. «James McIsaac, illustre et inconnu (18891970) ». Canadian Children's Literature/Littérature canadienne pour la jeunesse (64) : 26-39.

. 2000. Histoire de la littérature pour la jeunesse (Québec et francophonies du Canada) suivie d'un Dictionnaire des auteurs et des illustrateurs. Ottawa, David. 826 p.

. 2003a. Préface. In Délices de la littérature enfantine/ Children's Literature Feast of Titles. Ottawa, Institut canadien de microreproductions historiques : 17-22.

. 2003b. «L'image dans l'album pour enfants : enquête sur une libération». In La Littérature pour la jeunesse, 19702000, sous la direction de Françoise Lepage. Montréal, Fides : 21-44.

. 2003c. Paule Daveluy ou la Passion des mots. Montréal, Pierre Tisseyre. $285 \mathrm{p}$.

Michon, Jacques (sous la direction de). 2004. Histoire de l'édition littéraire au Québec. Vol. 2 : Le temps des éditeurs, 1940-1959. Montréal, Fides. 538 p.

Sarrasin, Francine. 1991. La Griffe québécoise dans l'illustration du livre pour enfants. Montréal/Québec, CommunicationJeunesse/Musée de la civilisation. 63 p.

Turgeon, Pierre. 1970. «Les illustrateurs Odette et Rodolphe Vincent : le temps des belles images est révolu». Perspectives (13 septembre), $\mathrm{n}^{\circ} 3$ (37) : 6-8.

Vincent, Josée. 2001. «Les associations d'éditeurs au Québec : de la théorie des associations à une étude de cas ». In Les Mutations du livre et de l'édition dans le monde du XVIII siècle à l'an 20oo, sous la direction de Jacques Michon et Jean-Yves Mollier. Montréal/Paris, Les Presses de l'Université Laval/L'Harmattan, p. 544-554. 